\title{
Die Bogenaustragung des spätgotischen Sternrippengewölbes im Chor der Kirche St. Peter und Paul in Owingen
}

\author{
Das Prinzipalbogenmodell*
}

VON RUDOLF BRÄNDLE

Im vorliegenden Beitrag wird untersucht und nachgewiesen, ob beim Bau des spätgotischen Sternrippengewölbes im Chor der Kirche St. Peter und Paul in Owingen die Entwurfsprinzipien des Prinzipalbogenmodells Anwendung fanden.

\section{1) Das Schema des Prinzipalbogens und der Stand der Forschung}

Durch die Zufälle der Überlieferung sind aus der Zeit der Spätgotik bis Ende des 15. Jahrhunderts wenige Bauskizzen über die Bogenaustragung von Zierrippengewölben erhalten. Dabei handelt es sich um Skizzen auf Pergament, Papier oder um Ritzzeichnungen in Stein.

In der Herzog August Bibliothek Wolfenbüttel ist eine Skizze auf Papier (Größe $29 \mathrm{~cm}$ x $21 \mathrm{~cm}$, undatiert, Zuordnung Ende des 15. Jahrhunderts) aus dem Musterbuch des Hans Hammer überliefert. ${ }^{1}$ Diese Skizze, deren Bedeutung François Fuchs zuerst erkannt und veröffentlicht hat, ${ }^{2}$ ist in der Abbildung 1 wiedergegeben. Im oberen Teil ist ein rechteckiger Grundriss dargestellt, in welchen die Rippenanordnung projiziert ist. Wenn keine gewundenen Reihungen - wie in diesem Fall - auftreten, ist dies eine Anordnung von Strecken. Aus dem oberen Teil wurde der Verlauf von Rippen entnommen und im unteren Teil als Abszisse aneinandergereiht. Mit diesen Aneinanderreihungen als Radius wurden Viertelkreisbögen gezogen. Die Ordinaten der Punkte dieser Kreisbögen sind dabei die Rippenverläufe in der dritten Dimension. Der Viertelkreis enthält so fast alle Informationen über den Verlauf der Rippen. Diese Überlieferung ist so wichtig, weil zu einem gegebe-

* Beim katholischen Pfarramt Owingen möchte ich mich für die Genehmigung zur Ausführung der Messungen im Chor der Kirche St. Peter und Paul bedanken. Gespräche mit dem Mesner über die Baugeschichte der Kirche waren hilfreich. Mein besonderer Dank gilt der Herzog August Bibliothek Wolfenbüttel für die freundliche Genehmigung der Reproduktion einer Abbildung aus dem Musterbuch des Hans Hammer.

1 Herzog August Bibliothek Wolfenbüttel: Cod. Guelf. 114.1 Extrav., fol. 23v.

2 François Fuchs, Introduction au Musterbuch de Hans Hammer, in: Bulletin de la Société des Amis de la Cathédrale de Strasbourg 20, 1992, S. 11-70, hier S. 47. 


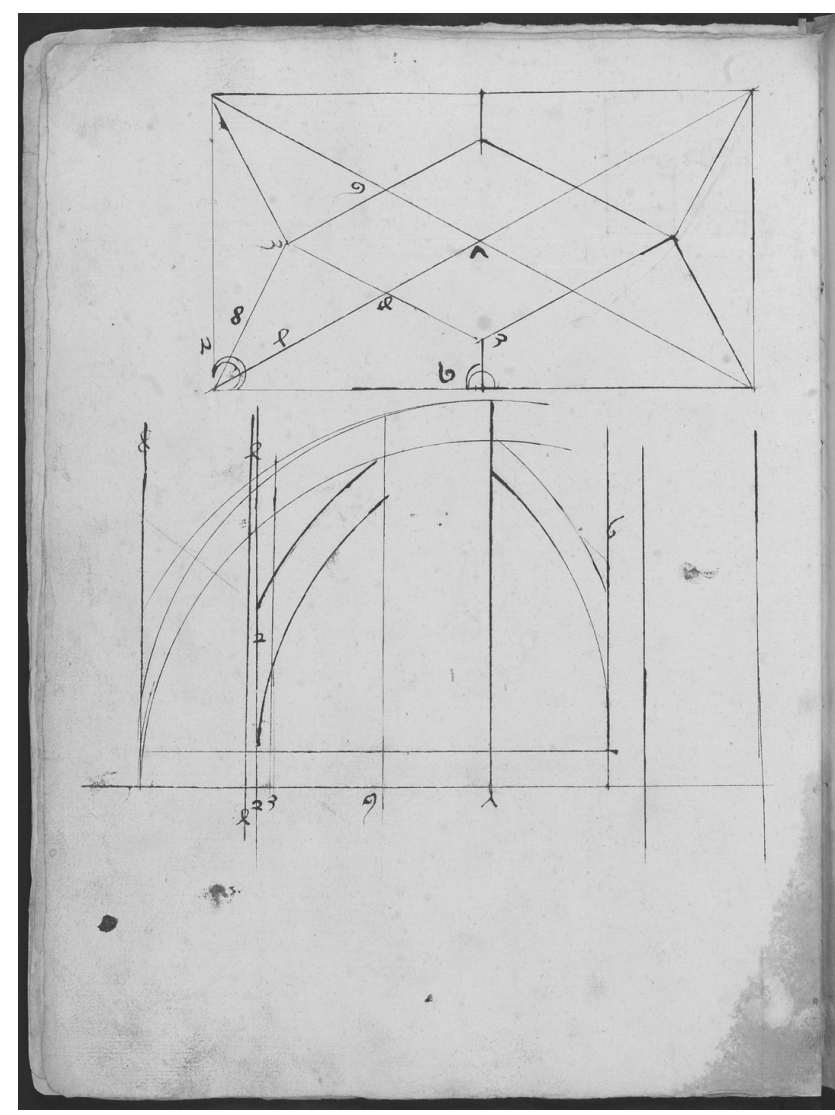

Abb. 1: Bauskizze über die Bogenaustragung von Zierrippengewölben aus dem Musterbuch des Hans Hammer. Quelle und Foto: Herzog August Bibliothek Wolfenbüttel: Cod. Guelf. 114.1 Extrav., fol. 23v.

nen Grundriss die dazu gehörige Bogenaustragung konstruiert wurde. Bei dieser Skizze handelt es sich wahrscheinlich um eine Studie mit verschiedenen Variationen des Entwurfes der Bogenaustragung, welche nicht in die Wirklichkeit umgesetzt wurde. Obwohl diese überlieferte zeitgenössische Skizze wohl kaum vollständig entschlüsselt werden kann, sind doch - trotz eventueller Formveränderungen des Papiers etc. - die Grundprinzipien gut erkennbar:

a) Die Bögen der Rippenverläufe (Unterseite der Rippen) sind Kreisbögen.

b) Der Radius der Kreisbögen ist möglichst gleich groß.

c) Es sind auch Knicke in der Rippenführung in der dritten Dimension vorgesehen.

Die Aussage dieser Skizze sollte wegen deren prinzipieller Bedeutung noch genauer untersucht und dargestellt werden. 
Indirekt ist in Friedrich Hoffstadts Gotisches ABC Buch ein altes Manuskript aus dem Barock (Skizzen mit Text aus dem Jahre 1695) überliefert. ${ }^{3}$ Der Autor Bertel Ranisch führt hier den terminus technicus, Gewölbter Prinzipal' ein, ${ }^{4}$ aus welchem sich dann der Begriff Prinzipalbogen entwickelt hat. Bertel Ranisch beschreibt, wie zur Konstruktion des Prinzipalbogens die Grundrissprojektionen möglichst langer vom Anfänger zum Scheitel führender Rippenzüge - für einige spezielle Fälle - aneinander gereiht werden. Die überlieferte Darstellung hat Modellcharakter, wie es der Untertitel dieses Beitrags zum Ausdruck bringt.

Das Spezifische beim Prinzipalbogenmodell ist, dass beim Entwurf in der Ebene (Papier, Pergament, Ritzzeichnung) die Bogenaustragung in der dritten Dimension fast gänzlich mitbestimmt wird. Für bestimmte Grundrisse, gewünschte Gewölbehöhen etc. sind die Zierformen so schon vorgegeben. Die entstandenen Zierformen tragen zur Statik des Gewölbes nichts Wesentliches bei.

Der Architekt Carl Meckel hat in einer grundlegenden Untersuchung über die graphische Konstruktion der Bogenaustragung spätgotischer Rippengewölbe aus der Grundrissprojektion des Rippenverlaufes viele noch erhaltene Gewölbe dargestellt. ${ }^{5}$ Darunter ist auch das große Sterngewölbe im Chor der Kirche St. Peter und Paul in Owingen, Nähe Überlingen am Bodensee. Den Zeichnungen ist ein Maßstab zugeordnet, aus dem die theoretisch geometrisch ermittelten Gewölbehöhen entnommen werden können. Im Text zu den Zeichnungen erwähnt Meckel keine Messungen, so dass er Theorie und Wirklichkeit nicht vergleichen konnte. Seine Grundrissprojektion der Rippenanordnung konnte von mir jedoch messtechnisch - s.u. Paragraph 4 - bestätigt werden. Wie Meckel selbst seine Ergebnisse verifiziert hat, ist unklar.

Werner Müller und Norbert Quien haben viele überlieferte Bauskizzen virtuell in die dritte Dimension übertragen und sich um eine allgemeine Betrachtungsweise verdient gemacht. ${ }^{6}$ Aus den Gewölberesten des spätgotischen Kreuzganggewölbes des Klosters Hirsau hat Jos Tomlow mit Hilfe des

3 Friedrich Hoffstadt, Gotisches ABC Buch. Das ist: Grundregeln des gotischen Styls für Künstler und Werkleute, Frankfurt a.M. 1840-1845.

4 Ebd., S. 169.

5 Carl Meckel, Die Konstruktion der figurierten Gewölbe in der deutschen Spätgotik, in: architectura 1, 1933, S. 107-114, hier S. 109.

6 Werner Müller, Grundlagen gotischer Bautechnik. Ars sine scientia nihil, München 1990, S. 155; ders. u. Norbert Quien, Von deutscher Sondergotik. Architekturphotographie, Computergraphik, Deutung (Saecula Spiritualia, Bd. 33), Baden-Baden 1997; dies., Spätgotik virtuell. Für und Wider die Simulation historischer Architektur, Weimar 1999; dies., Erdachte Formen, errechnete Bilder. Deutschlands Raumkunst der Spätgotik in neuer Sicht, Weimar 2000; dies., Böhmens Barockgotik. Architekturbetrachtung als computergestützte Stilkritik, Weimar 2000; dies., Virtuelle Steinmetzkunst der Österreichischen und Böhmisch-Sächsischen Spätgotik. Die Gewölbeentwürfe des Codex Miniatus 3 der Österreichischen Nationalbibliothek in Wien (Studien zur internationalen Architekturund Kunstgeschichte, Bd. 37), Petersberg 2005. 
Prinzipalbogenmodells die Bogenaustragung theoretisch rekonstruiert. ${ }^{7}$ Beim längst abgetragenen Westflügel des spätgotischen Kreuzganges des Klosters Alpirsbach ist er ähnlich vorgegangen. ${ }^{8}$

Norbert Nußbaum und Sabine Lepsky führen Carl Meckels theoretische Bogenaustragung des Chorgewölbes in der Kirche St. Peter und Paul in Owingen ohne Maßstab kommentarlos an. ${ }^{9}$ Sie bilden außerdem eine Ritzzeichnung an der Langhauswand (vor 1509, Originalmaßstab 1:1) der Kirche St. Sigismund in Sydlowiec in Polen ab, welche - soweit aus der Abbildung erkennbar - die gleiche Rippenführung wie das Chorgewölbe in Owingen aufweist. Obwohl die Hilfslinien für die Konstruktion der Bogenaustragung in der Ritzzeichnung spärlich sind, kann man links unten in der Skizze einen längeren Kreisbogen erkennen, worauf in Paragraph 5 eingegangen wird.

Ute Germund führt auch die Meckelsche Bogenaustragung im Chor der Kirche in Owingen an und sieht die Problematik der spätgotischen Rippenkonstruktionen darin, dass die Methoden anhand von Rissen mit Lehr- und Modellcharakter entwickelt worden sind. ${ }^{10}$

Ob Modellvorstellungen wie das Prinzipalbogenmodell oder ein anderes Modell des Entwurfes mit der Wirklichkeit erhaltener spätgotischer Rippengewölbe vereinbar sind, muss von Fall zu Fall untersucht und entschieden werden. Hierbei ist noch viel zu tun, denn nur durch Vergleich von Messungen an erhaltener Bausubstanz mit Entwurfsmodellen lassen sich Erkenntnisse über spätgotische Bautechnologie gewinnen.

\section{2) Kurze Baugeschichte von St. Peter und Paul in Owingen und spätere Veränderungen}

Über einen etwaigen Vorgängerbau an gleicher Stelle ist nichts bekannt. Nach dem Kirchenführer ist die Kirche etwa um das Jahr 1498 von der Deutschordenskommende Mainau erbaut worden, worauf auch die Jahreszahl über dem Südportal hinweist. ${ }^{11}$ Das Rippengewölbe im Chor soll etwa in der gleichen Zeit von Heinrich Klingenberg errichtet worden sein. Das ganze Ge-

7 Jos Tomlow, Versuch einer (zeichnerischen) Rekonstruktion des Gewölbes im spätgotischen Kreuzgang des Klosters Hirsau, in: Hirsau. St. Peter und Paul 1091-1991. Zur Archäologie und Kunstgeschichte (Forschungen und Berichte der Archäologie des Mittelalters in Baden-Württemberg, Bd. 10/1), Stuttgart 1991, S. 365-387.

8 Jos Tomlow, Neue Erkenntnisse zur Baugeschichte des Kreuzganges. Entwurf und Ausführung eines mittelalterlichen spätgotischen Gewölbes, in: Alpirsbach. Zur Geschichte von Kloster und Stadt (Forschungen und Berichte der Bau- und Kunstdenkmalpflege in Baden-Württemberg, Bd.10/1), Stuttgart 2001, S. 427-448.

9 Norbert Nußbaum u. Sabine Lepsky, Das gotische Gewölbe. Eine Geschichte seiner Form und Konstruktion, München 1999, S. 179.

10 Ute Germund, Konstruktion und Dekoration als Gestaltungsprinzipien im spätgotischen Kirchenbau. Untersuchungen zur mittelrheinischen Sakralbaukunst (Manuskripte zur Kunstwissenschaft in der Wernerschen Verlagsgesellschaft, Bd. 53), Worms 1997, S. 67.

11 Kirchenführer der Pfarrgemeinde Owingen mit Bilafingen, Freiburg 2001. 
bäude dürfte auf freiem Platz erstellt worden sein, sodass keine baulichen Randbedingungen auftraten. Über bauliche Veränderungen im Chorbereich ist nichts bekannt. Abbildung 2 zeigt einen Blick in das Chorgewölbe. Der sternförmige Charakter der Rippenanordnung ist leicht zu erkennen.

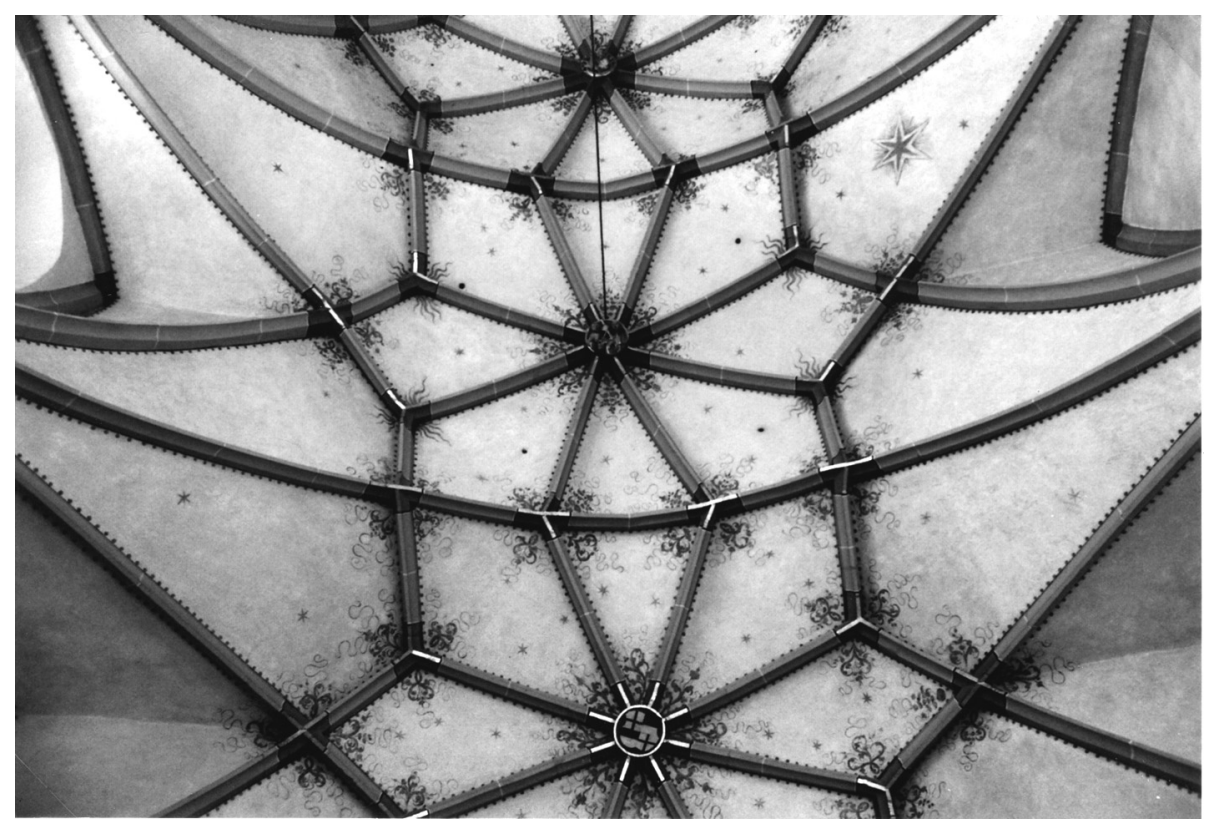

Abb. 2: Blick in den Scheitel des Sternrippengewölbes im Chor. Foto: Rudolf Brändle.

\section{3) Die theoretische Bogenaustragung nach dem Prinzipalbogenmodell}

Aus Platzgründen sind in der Abbildung 3 bei der Projektion des Rippenverlaufes in den Grundriss nur der 3/8-Chorschluss und eines der drei queroblongen Joche dargestellt. Ohne gewundene Reihungen ergibt sich ein Netz aus Strecken. Da die Chorseiten gleich lang sind und diese paarweise einen Winkel von $135^{\circ}$ einschließen, muss zur Konstruktion des Grundrisses der 3/8-Chorschluss zu einem regelmäßigen Oktogon erweitert werden, was zur Lichten Weite LW des Gewölbes und zur Jochtiefe führt. Nach dem Prinzipalbogenmodell werden die in den Grundriss projizierten Rippenstücke von Anfänger A (s. Abbildung 3, links oben) auf dem längsten Wege zum Scheitel E aneinandergereiht. Dazu wird zuerst der Verlauf der Diagonalrippe von $A$ aus über $C$ hinaus verlängert. Dann wird noch die fehlende Strecke CE angereiht. Dazu wird um C auf der Diagonalrippe der Kreis mit Radius CE gezogen, welcher die Verlängerung von $\mathrm{AD}$ in $\mathrm{M}$ schneidet. Um diesen Mittelpunkt $\mathrm{M}$ wird ein Viertelkreis (s. Abbildung 3, dunkle Schattierung) mit dem Radius AM gezogen. Der fallende Prinzipalbogen über dem gebrochenen Rippenzug hat den Radius $\mathrm{R}_{\mathrm{Pr}_{\mathrm{r}}}=\mathrm{AM}=\mathrm{ME}^{\prime}$ und ist für alle Rippenstücke 


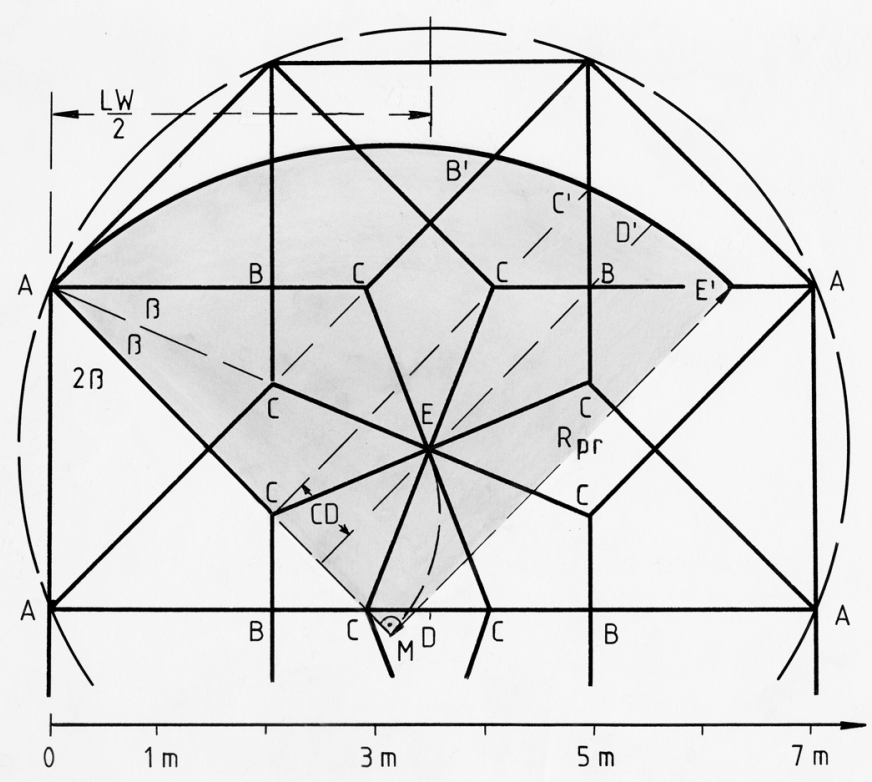

Abb. 3: In den Grundriss projizierte Rippenanordnung des Chorgewölbes, LW = Lichte Weite, $4 \times \beta=$ rechter Winkel, Viertelkreisfläche des Prinzipalbogens (mit $R_{P_{r}}=$ Prinzipalbogenradius) dunkler gekennzeichnet. Realisation und Foto: Rudolf Brändle.

gleich. Die Gewölbehöhen ergeben sich mit $\mathrm{B}^{\prime}$ über $\mathrm{B}, \mathrm{C}^{\prime}$ über $\mathrm{C}$ und $\mathrm{E}^{\prime}$ über E. Da die Projektion der Gurtrippe von A aus über B und C nach D kürzer als die Strecke AM ist, liegt $\mathrm{D}^{\prime}$ unterhalb von $\mathrm{E}^{\prime}$. Um $\mathrm{D}^{\prime}$ zu ermitteln, wird dann an die Verlängerung der Diagonalrippe $\mathrm{AC}$ in $\mathrm{C}$ die Strecke $\mathrm{CD}$ angetragen, womit sich $\mathrm{D}^{\prime}$ ergibt.

Bei dieser Konstruktion wurden zuerst die Rippenpunkte im Scheitel ( $\left.E^{\prime}\right)$ auf gleicher Höhe festgelegt. Die Tangenten an die Rippen im Scheitel sind horizontal. Dann wurden die weiteren Rippenpunkte mit fallendem Prinzipalbogen ermittelt. Da die Rippenzüge in diesem Falle vom Scheitel zum Anfänger alle gleich lang sind, liegen die Anfänger auf gleicher Höhe. Die Tangenten an die Rippen im Anfänger sind vertikal. Die Rippen entspringen am Anfänger ohne Knick (mathematisch: stetig) aus der Wand.

Veranschaulicht wird diese Konstruktion in der dritten Dimension, wenn die Zeichenunterlage entlang $\mathrm{CM}$ und $\mathrm{ME}^{\prime}$ und dem Viertelkreisbogen von den Punkten $E^{\prime}$ über D', B' bis A aufgeschnitten wird. Entlang der Diagonalrippe $\mathrm{AC}$ wird im rechten Winkel aufgebogen und um $\mathrm{CC}^{\prime}$ gefaltet, bis $\mathrm{M}$ an den Ort von B rückt. In der Abbildung 4 ist dies räumlich gut zu erkennen. Dem Leser sei empfohlen, mit einer Papierkopie der Abbildung 3 gleich zu verfahren. 


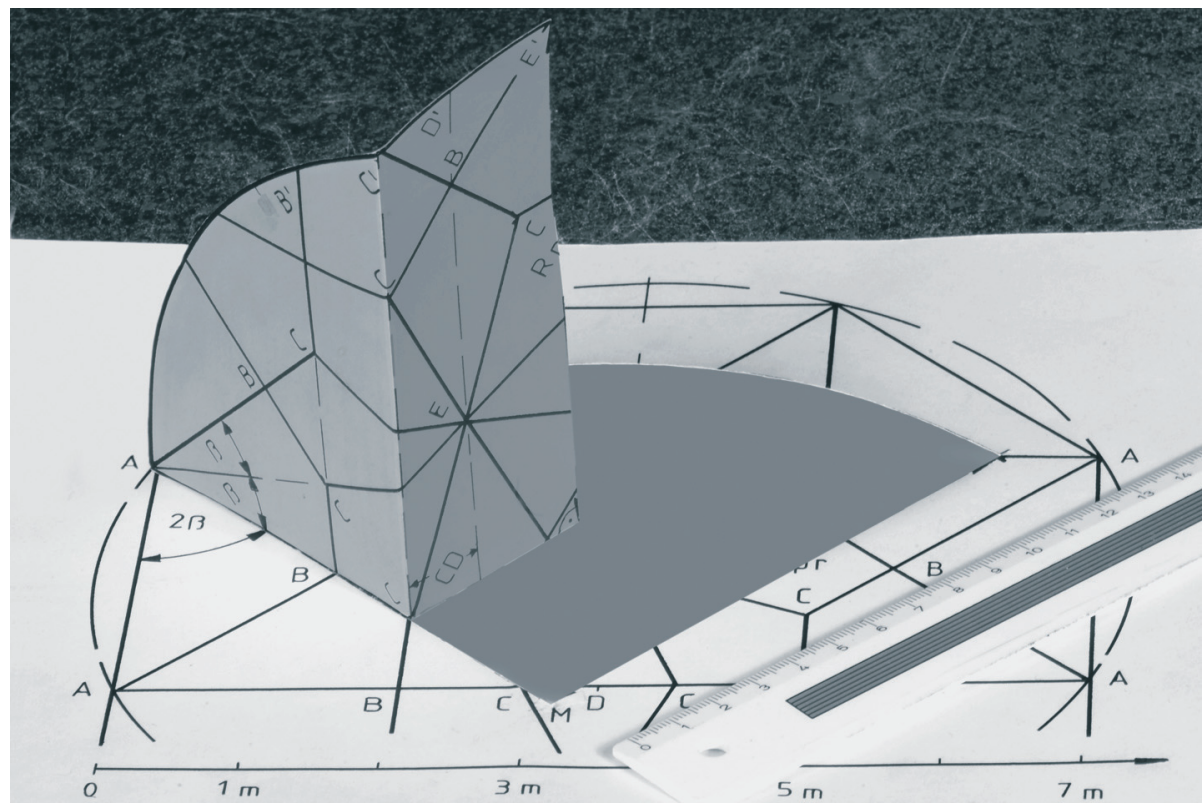

Abb. 4: Veranschaulichung in der dritten Dimension: Die projizierte Rippenanordnung der Abbildung 3 teilweise aufgeschnitten, aufgebogen und teilweise gefaltet. Realisation und Foto: Rudolf Brändle.

Von jedem Anfängerpunkt A aus gibt es vier gleichwertige Möglichkeiten durch Aneinanderreihung von Rippenprojektionen den Prinzipalbogen zu konstruieren. In allen diesen Fällen werden die gleichen Rippenzüge durchlaufen und damit ist der Radius aller Rippenbögen $\mathrm{R}_{\mathrm{Pr}}=$ konstant. Um zeichnerische Ungenauigkeiten bei der graphischen Konstruktion auszuschließen, wurden mit Hilfe ebener Trigonometrie die relevanten Gewölbehöhen gemäß der Konstruktion in algebraische Gleichungen entwickelt. Der Übersicht halber werden die darin enthaltenen Konstanten durch Zahlenwerte ersetzt wiedergegeben:

$$
\begin{array}{ll}
\mathrm{BB}^{\prime}=\mathrm{f}(\mathrm{LW})=0,527 \mathrm{LW} & \mathrm{CC}^{\prime}=\mathrm{g}(\mathrm{LW})=0,593 \mathrm{LW} \\
\mathrm{DD}^{\prime}=\mathrm{h}(\mathrm{LW})=0,621 \mathrm{LW} & \mathrm{R}_{\mathrm{Pr}}=\mathrm{EE}^{\prime}=\mathrm{ME}^{\prime}=\mathrm{i}(\mathrm{LW})=0,638 \mathrm{LW}
\end{array}
$$

Es sind lineare Gleichungen mit der einzigen unabhängigen Variablen LW (Lichte Weite) des Gewölbes.

Für die Ausführung der Berechnungen genügte ein handelsüblicher programmierbarer grafikfähiger elektronischer Taschenrechner. Mit den eingespeicherten theoretischen Funktionen konnte dieser an den Ort der Messungen mitgenommen werden, wobei schon während der Messungen Vergleiche mit den theoretischen Werten möglich waren. 
In der in Abbildung 1 dargestellten Skizze aus dem Musterbuch des Hans Hammer treten Knicke in der Rippenführung in der dritten Dimension auf, was als eine Variation des Entwurfes gedeutet werden kann. Dies ist nicht zwingend notwendig, weil es sich auch nach dem Prinzipalbogenmodell im engeren Sinne nicht ergibt. In der theoretischen Bogenaustragung im untersuchten Falle wurde kein solcher Knick vorgesehen.

\section{4) Die Messungen}

Für die Messungen wurde ein auf ein handelsübliches Stativ montierter Laserentfernungsmesser GEO-300 der Firma Jenoptik im Einpersonenbetrieb verwendet. Die Steine des Gewölbes sowie die darauf angebrachte Farbe zeigten ein für Laserlicht von $904 \mathrm{~nm}$ ausreichendes Reflexionsverhalten. Jede gewünschte Einzelmessung konnte ausgeführt werden.

Die Messungen ergaben, dass es sich um einen 3/8-Chorschluss handelt, da die drei Seiten gleich lang sind und diese jeweils paarweise einen Winkel von $135^{\circ}$ einschließen. Es zeigte sich außerdem, dass die Rippenanfänger in etwa auf einer horizontalen Ebene liegen. Die Schlusssteine und sich entsprechende Gewölbehöhen in den drei Jochen und im 3/8-Chorschluss liegen angenähert auf parallelen Linien, welche keinen Winkel mit der horizontalen Ebene einschließen. Vereinfachend kommt in diesem Falle hinzu, dass am Kämpfer die Rippen ohne Knick (mathematisch: stetig) aus der Wand entspringen und dort deshalb die Tangenten an die Rippen vertikal sind. Insgesamt lässt sich also feststellen, dass die Auswahl dieses Messobjektes sich bezüglich Symmetrie und Homogenität in den Jochen als günstig erwies.

Die Vorgaben Meckels bezüglich der Projektion des Rippenverlaufes konnten bestätigt werden. Bezüglich der aus der Meckelschen Skizze entnommenen und maßstäblich hochgerechneten Lichten Weite ergab sich $\mathrm{LW}_{\text {Meckel }}=7,5 \mathrm{~m}$, während meine gemessene und gemittelte Lichte Weite etwa $\mathrm{LW}=7,1 \mathrm{~m}$ beträgt.

Die relevanten Einzelmessungen der Gewölbehöhen in den drei Jochen des Chores und im 3/8-Chorabschluss wurden ausgeführt. Einige Einzelmessungen im 3/8-Chorabschluss konnten nicht ausgeführt werden, da der Hochaltar die optische Sicht vom Messgerät zu den Messpunkten versperrt. Die Messungen in den drei Jochen wurden zum Mittelwertjoch gemittelt. Diese Mittelung ist nur sinnvoll, da sich keine größeren prozentualen Abweichungen - vielleicht in zunehmendem oder abnehmendem Sinne - ergeben. So bezieht sich diese Aussage auf das ganze Chorgewölbe und nicht nur auf ein einzelnes, vielleicht idealisiertes, Einzeljoch.

Bei den vorhandenen Bogenlängen von bis zu $7 \mathrm{~m}$ mit einem Bogenradius von über $4 \mathrm{~m}$ bot es sich außerdem an, den geometrischen Ort der Unterseite der Rippen direkt auszumessen und aufzuzeichnen, um daraus die Krümmung zu entnehmen. Nur bei den in Abbildung 3 dargestellten Gurtrippenbögen vom Punkt A aus über B nach $\mathrm{C}$ zwischen dem dritten Joch und 
dem anschließenden 3/8-Chorschluss ist dies wegen des notwendigen Standortes des Messgerätes einigermaßen möglich. Dasselbe gilt für die Diagonalbögen vom gleichen Punkt $A$ aus bis $\mathrm{C}$.

Bei meiner Untersuchung der spätgotischen Gewölbe des Kreuzganges des Klosters Alpirsbach war diese Direktmessung wegen kleiner Dimensionen zu ungenau und nicht verwertbar. ${ }^{12}$

\section{5) Ergebnis: Vergleich Theorie und Wirklichkeit}

Theorie: Mit der gemessenen Lichten Weite des Gewölbes von 7,1 m ergeben sich aus dem Prinzipalbogenmodell (Nomenklatur s. Abbildung 3) die Gewölbehöhen: $\mathrm{BB}^{\prime}=3,74 \mathrm{~m}, \mathrm{CC}^{\prime}=4,21 \mathrm{~m}, \mathrm{DD}^{\prime}=4,40 \mathrm{~m}$ und der Einheitsradius $R_{\mathrm{Pr}}=$ konst. $=4,53 \mathrm{~m}$.

Wirklichkeit: Die zum Mittelwertjoch gemittelten Einzelmessungen in den drei Jochen und die Messungen im 3/8-Chorschluss weichen von den theoretischen Werten um $+/-6 \%$ ab. Direktmessungen des geometrischen Ortes der Unterseite der Rippen (Abszisse und Ordinate von Punkten) waren mit brauchbarer Genauigkeit nur bei vier längeren Rippenbögen möglich, wobei sich der Krümmungsradius mit 4,4 $\mathrm{m}+/-15 \%$ ergab. Dessen relativ kleine Abweichung vom theoretischen Wert würde auch bei der geringen Gewichtung von vier Einzelmessungen gegen die Vielzahl der indirekten Messungen nicht ins Gewicht fallen.

Durch die gute Übereinstimmung von Theorie und Wirklichkeit ergibt sich, dass kein Knick in der dritten Dimension vorhanden sein kann. Mit dem Prinzipalbogenmodell im strengeren Sinne oder einem ähnlichen Verfahren ist das Chorgewölbe entworfen worden.

Der einheitliche Krümmungsradius der Rippen ist - wie in der Theorie verifiziert worden. Einige mögliche Direktmessungen weisen in die gleiche Richtung. Wenn, theoretisch angenommen, Rippen mit zwei deutlich verschiedenen Krümmungen versetzt worden wären, würde die Übereinstimmung von Theorie und Wirklichkeit nicht so gut sein. Die Bautechnologie des späten Mittelalters war wohl auch nicht in der Lage mit nur um wenige Prozente verschiedenen Bogenradien zu arbeiten. Für das untersuchte Gewölbe waren wohl auch keine baulichen Zwänge bei der Erstellung vorhanden, wie erwähnt war nicht auf einen Vorgängerbau Rücksicht zu nehmen.

Der aus der Meckelschen Skizze entnommene Wert der Lichten Weite $\mathrm{LW}_{\text {Meckel }}=7,5 \mathrm{~m}$ führt mit den theoretischen Berechnungen des Prinzipalbogenmodells auf einen Radius von $\mathrm{R}_{\text {PrMeckel }}=4,8 \mathrm{~m}$, was etwa dem Wert in seiner Skizze entspricht.

In der von Norbert Nußbaum und Sabine Lepsky veröffentlichten Ritzzeichnung in St. Sigismund in Sydlowiec lässt sich die Konstruktion der

12 Rudolf Brändle, Die Bogenaustragung der spätgotischen figurierten Rippengewölbe des Kreuzganges Kloster Alpirsbach. Das Prinzipalbogenmodell, in: architectura 35, Bd. 2, 2005, S. 138-147. 
Bogenaustragung kaum erkennen. Der Bogenradius des längeren abgebildeten Kreisbogens lässt sich jedoch in etwa entnehmen. Das Verhältnis Bogenradius/Lichte Weite im Vergleich ergibt:

(Bogenradius/Lichte Weite) $)_{\text {Ritzzeichnung }}=0,64$

(Bogenradius/Lichte Weite) $)_{\text {Chorgewölbe Owingen }}=0,638$

Die Übereinstimmung ist so gut, dass für beide Entwürfe aus derselben Grundstruktur die gleichen oder ähnlichen Prinzipien wirksam waren.

\section{6) Zusammenfassung}

Dem zeitgenössischen Musterbuch des Hans Hammer wurde eine Skizze zur Bogenaustragung eines spätmittelalterlichen Rippengewölbes über rechteckigem Grundriss entnommen, um das Verfahren der Konstruktion auf ein anderes, noch bestehendes Gewölbe anzuwenden. Das untersuchte spätgotische Chorgewölbe der Kirche St. Peter und Paul in Owingen wurde Ende des 15. Jahrhunderts - wahrscheinlich auf freiem Gelände - erbaut. Eine Überlieferung der Bogenaustragung dazu gibt es nicht. Jedoch zeigt die oben erwähnte Ritzzeichnung an der Langhauswand in Sydlowiec die, soweit aus der Abbildung feststellbar, gleiche Projektion der Rippenanordnung in den Grundriss.

Die Bogenaustragung des untersuchten Gewölbes wurde von Meckel untersucht und dessen Ergebnisse wurden von anderen Autoren oft angeführt. Von umfangreichen Vermessungen wird nichts berichtet und deshalb haben diese Aussagen eher theoretischen Charakter.

Bei meinen berührungslosen Messungen mit moderner Lasertechnik stellte sich heraus, dass das Chorgewölbe bezüglich Symmetrie und Homogenität in den drei Jochen bis heute gut erhalten ist. Da dies die Grundvoraussetzung für aussagefähige Vermessungen ist, eignet sich dieses Objekt für umfangreiche Messungen. Zuerst wurde aus der Dimensionierung des Chores der 3/8-Chorschluss messtechnisch nachgewiesen. Die relevanten Gewölbepunkte wurden berührungslos ausgemessen. Die Messwerte in den drei Jochen wurden zum Vergleich mit den theoretischen Werten zum Mittelwertjoch gemittelt. So bezieht sich die Aussage nicht auf ein vielleicht idealisiertes Modelljoch sondern auf das ganze Gewölbe.

Zum theoretischen Entwurf mit dem Prinzipalbogenmodell muss in der Rippenprojektion des Rippenverlaufes in den Grundriss der 3/8-Chorschluss zum regelmäßigen Oktogon erweitert werden. Mit fallendem Prinzipalbogen (Gewölbescheitel alle gleich hoch, Tangenten an die Rippen dort horizontal) wurden mit dem Einheitsradius $\mathrm{R}_{\mathrm{Pr}}$ (Prinzipalbogen) über gebrochenem Rippenzug die relevanten Gewölbehöhen als lineare Funktion der Lichten Weite LW algebraisch berechnet. 
Ein Vergleich von Theorie (Modell) und Wirklichkeit zeigt, dass die gemessenen Werte um $+/-6 \%$ von den errechneten abweichen. Wenige davon unabhängige mögliche Direktmessungen der Rippenführung zeigen $+/-15 \%$ Abweichung von den theoretischen Werten. Die Übereinstimmung ist also unter den Umständen als gut anzusehen.

Das Verhältnis des Radius $\mathrm{R}_{\mathrm{Pr}_{\mathrm{r}}}$ des Prinzipalbogens zur Lichten Weite LW der Ritzzeichnung in St. Sigismund in Sydlowiec ist - soweit aus der Abbildung erkennbar - mit dem des Owinger Chorgewölbes fast identisch. Es müssen also in beiden Fällen die gleichen oder ähnliche Entwurfsprinzipien angewendet worden sein.

Es wurde folgendes nachgewiesen:

a) Der geometrische Ort der Unterseite der Rippen hat die Form von Kreisbögen verschiedener Bogenlänge.

b) Alle Rippenbögen weisen einen für das gesamte Gewölbe spezifischen einheitlichen Prinzipalbogenradius $\mathrm{R}_{\mathrm{Pr}}$ auf.

c) Die Projektion des Rippenverlaufes in den Grundriss beinhaltet somit alle Informationen für die Wölbung in der dritten Dimension. Die Wölbung wurde also bei der Konstruktion in der Zeichenebene vollkommen mitbestimmt.

Beim Entwurf der Grundrissprojektion des Rippenverlaufes musste bei aller ,künstlerischen Freiheit' die Erbauung mit bedacht werden. Mit berücksichtigt werden mussten die örtlichen Gegebenheiten wie die des Steinmaterials, der Logistik, wechselnde Steinmetzen etc. und nicht zuletzt die Kosten. So ist es nicht verwunderlich, dass auf Normierung wie z.B. gleiche Rippenkrümmung geachtet wurde. Die Rationalisierung muss nicht zu geringerer stilistischer Qualität führen. Dem Besucher der Kirche in Owingen bleibt es verborgen, dass die Grundgeometrie des Chorgewölbes auf einfache Bauausführung angelegt ist. Die relativ einfachen harmonischen Rippenanfänger und die Anordnung der Zierformen Richtung Scheitel führen zu leicht verspielter Eleganz.

Adresse des Verfassers: Dr. Rudolf Brändle, Ahornweg 10, 83714 Miesbach, E-Mail: braendle@kabelmail.de 
